\title{
Genetic variation and environmental effects on beta-conglycinin and glycinin content in Brazilian soybean cultivars
}

\author{
Mercedes Concórdia Carrão-Panizzi ${ }^{(1)}$, Prachuab Kwanyuen ${ }^{(2)}$, Sevim Zeynep Erhan ${ }^{(3)}$ \\ and Ivani de Oliveira Negrão Lopes ${ }^{(1)}$
}

\begin{abstract}
(1)Embrapa Soja, Rodovia Carlos João Strass, Distrito de Warta, CEP 86001-970 Londrina, PR, Brazil. E-mail: mercedes@cnpso.embrapa.br, negrao@cnpso.embrapa.br (2)North Carolina State University, Crop Science Department, Zip Code 27695 Raleigh, NC, USA. E-mail: prachuab-kwanyuen@nscu.edu (3)United States Department of Agriculture, Agricultural Research Service, National Center for Agricultural Utilization Research, Food and Industrial Oil Unit, Zip Code 61604 Peoria, IL, USA. E-mail: sevim.erhan@ars.usda.gov
\end{abstract}

\begin{abstract}
The objective of this work was to determine genetic and environmental effects on beta-conglycinin and glycinin content in Brazilian soybean cultivars. The concentrations of these protein fractions were analyzed by scanning densitometry after electrophoresis, in 90 Brazilian soybean cultivars sown in Ponta Grossa, PR, in 2001. The effects of the sowing location were determined in the cultivar MG/BR 46 (Conquista), sown in 16 locations of Goiás and Minas Gerais states (Central Brazil), and in the cultivar IAS 5, sown in 12 locations of Paraná and São Paulo states (Southern Brazil), in 2002 soybean season. A significant variability for beta-conglycinin (7S) and glycinin (11S) protein fractions ratio was observed among the 90 Brazilian soybean cultivars. 'MS/BRS 169' (Bacuri) and 'BR-8' (Pelotas) presented the highest and the lowest 11S/7S ratios (2.76 and 1.17, respectively). Beta-conglycinin protein fractions presented more variability than glycinin protein fractions. Grouping test classified 7S proteins in seven groups, $11 \mathrm{~S}$ proteins in four groups, and protein fraction ratios $(11 \mathrm{~S} / 7 \mathrm{~S})$ in nine groups. Significant effect of sowing locations was also observed on protein fractions contents. There is a good possibility of breeding for individual protein fractions, and their subunits, without affecting protein content.
\end{abstract}

Index terms: eletrophoresis, nutraceutic properties, protein fractions, seed, sowing location.

\section{Variação genética e ambiental e teores de beta-conglicinina e glicinina em cultivares de soja brasileiras}

Resumo - O objetivo deste trabalho foi avaliar os efeitos da variação genética e ambiental sobre os teores de beta-conglicinina e glicinina em cultivares de soja brasileiras. A concentração dessas frações protéicas foi determinada por densitometria após eletroforese, em 90 cultivares de soja, semeadas em Ponta Grossa, PR, em 2001. Os efeitos dos locais de semeadura foram determinados na cultivar MG/BR 46 (Conquista), semeada em 16 locais de Goiás e Minas Gerais, e na cultivar IAS 5, semeada em 12 locais no Paraná e em São Paulo, em 2002. Foi observada variabilidade significativa quanto à razão entre as frações protéicas da beta-conglicinina (7S) e da glicinina (11S), entre as 90 cultivares avaliadas, em que a 'MG/BR 169' (Bacuri) apresentou a maior razão $11 \mathrm{~S} / 7 \mathrm{~S}(2,76)$ e a 'BR-8' (Pelotas) a menor $(1,17)$. As frações protéicas de beta-conglicinina apresentaram maior varibilidade do que as de glicinina. A análise de agrupamento discriminou as proteínas $7 \mathrm{~S}$ em sete grupos, as $11 \mathrm{~S}$ em quatro, e as razões $11 \mathrm{~S} / 7 \mathrm{~S}$ em nove. Efeito significativo dos locais de semeadura também foi observado sobre os teores das frações protéicas. Existe uma boa possibilidade de melhoramento para as frações individuais de proteínas e suas subunidades, sem que o teor de proteína seja alterado.

Termos para indexação: eletroforese, propriedades nutracêuticas, frações protéicas, semente, local de semeadura.

\section{Introduction}

The major storage proteins of soybean seeds are beta-conglycinin (7S globulin) and glycinin (11S globulin). Beta-conglycinin is a trimeric protein composed of three subunits alpha', alpha and beta (Thanh \& Shibasaki, 1977). Glycinin is a hexameric protein composed of five subunits $\mathrm{A} 1 \mathrm{aB} 1 \mathrm{~b}, \mathrm{~A} 1 \mathrm{bB} 2$, $\mathrm{A} 1 \mathrm{~B} 1 \mathrm{a}, \mathrm{A} 3 \mathrm{~B} 4$ and $\mathrm{A} 5 \mathrm{~A} 4 \mathrm{~B} 3$, and each subunit is composed of acidic and basic polypeptides, which are linked together by a disulfide bond (Kitamura et al., 1976; Staswick et al., 1984). Protein fractions have different functional properties, related to gel formation, thermal stability and emulsification (Utsumi et al., 
1997). Heat and calcium-induced coagulum (tofu gel) made from high content glycinin (11S) soybean is harder than with higher $7 \mathrm{~S}$ fraction soybean (Saio et al., 1969). The large number of disulfide bonds $(-\mathrm{SH})$, due to more methionine and cystein in glycinin fraction, improves gel formation capacity (Yamauchi et al., 1981) making glycinin gel harder and more turbid than beta-conglycinin gel. However, the higher hydrophobicity, the easy unfolded structure, and less thermally stable properties of beta-conglycinin make its emulsifying ability stronger than that of glycinin (Fukushima, 2001).

Genetic studies revealed variation on protein fractions and subunits, and cultivars with high levels of $7 \mathrm{~S}$ or $11 \mathrm{~S}$ fractions are available (Tsukada et al., 1986; Takahashi et al., 2000; Ogawa et al., 1989). The cultivar Tohoku 124 presents high ratio of glycinin to beta-conglycinin (Takahashi et al., 2000), while the cultivar Kyu-kei 305 contains only glycinin as storage proteins and lacks the three major allergenic proteins of $28 \mathrm{~K}$, alpha and beta subunits (Takahashi et al., 2000). Kyu-kei is the cultivar with the least quantities of allergens. Protein contents of these cultivars were the same as the recurrent parents. This tendency was also observed by Harada et al. (1983), who reported no correlation between $11 \mathrm{~S} / 7 \mathrm{~S}$ ratio and seed protein content, observing no significant differences in protein content among three genotypes. Yaklich (2001), however, reported that high-protein lines appear to contain more beta-conglycinin and glycinin than normal-protein soybean lines, and the amount of subunits and polypeptides was different among lines. These conclusions were observed from data of several breeding lines evaluated for five years. Moraes et al. (2006) observed, in two high protein soybean isolines, increasing of $11 \mathrm{~S}$ proteins and no changes in $7 \mathrm{~S}$ proteins, which consequently promoted higher $11 \mathrm{~S} / 7 \mathrm{~S}$ ratio.

Environmental effects on these protein fractions have been reported (Murphy \& Ressureccion, 1984; Fehr et al., 2003). In a study of interaction between genotype and environment, Fehr et al. (2003) observed significant differences among all traits (protein fractions), except for the A3 subunit of glycinin, in 14 genotypes grown in 8 locations for three years. They observed no significant interactions between genotypes and years or locations. Murphy \& Ressureccion (1984) reported differences for glycinin contents within years.
Helms et al. (1998) found a genotype x environment interaction for protein content and $11 \mathrm{~S} / 7 \mathrm{~S}$ ratio. The magnitude of the differences between the two cultivars for $11 \mathrm{~S} / 7 \mathrm{~S}$ ratio varied among locations within year.

Soybean proteins have also been claimed to be effective in the prevention of cardiovascular diseases (USFDA, 1999), and beta-conglycinin (7S) has a role in the upregulation of liver high-affinity LDL receptors (Lovati et al., 1998). Duranti et al. (2004), investigating the effect of daily administration of isolated soybean 7S globulin alpha', observed a significant reduction on plasma cholesterol and triglycerides levels in hypercholesterolemic rats. The same authors showed that it is possible to enhance the lipid lowering effects by further increases in the alpha' subunit doses. Estimated amounts of alpha' subunit in the 7S globulin trimer is about one third of the total globulin weight (Duranti et al., 2004).

In Brazil, soybean is grown in a wide range of environments, and differences for protein fractions among Brazilian cultivars have not been analyzed. Information about differences in the amount of protein fractions among soybean cultivars could aim at processors interested in specific products, which need emulsifying or gel formation properties. Those information could also be interesting to process functional foods, since there is a promise of biological effects on lipid-lowering therapy.

The objective of this work was to determine genetic and environmental effects on beta-conglycinin - 7S (alpha', alpha, and beta subunits) and glycinin - 11S (acidic and basic subunits) contents in Brazilian soybean cultivars.

\section{Materials and Methods}

In a first experiment, 90 Brazilian soybean cultivars were analyzed to determine genetic differences on contents of protein fractions. They were sown in plots of 4 rows ( $5 \mathrm{~m}$ length), in fertile soil, in Ponta Grossa, Paraná state $\left(25^{\circ} 5^{\prime} \mathrm{S}\right)$, in 2001 crop season.

In a second experiment, effects of the sowing location were determined in the cultivar MG/BR 46 (Conquista), sown in 16 locations (Anápolis, Cerrados, Senador Canedo, Alvorada, Cristalina-1, São Miguel do Passa Quatro, Rio Verde, Uberaba, Conquista, Uberlândia, Luziana, Sacramento, Buritis, Iraí de Minas, Cristalina-2 and Chapadão do Céu) of Goiás 
and Minas Gerais states (Central Brazil), in 2002 crop season. Cristalina-1 and Cristalina-2 locations are different fields of the same location, and it was observed a high infestation of nematodes in the field Cristalina-2. Effects of the sowing location was also determined in cultivar IAS 5, sown in 12 locations (Guaíra, Pedrinhas Paulista, Pirassununga, Londrina, Morro Agudo, Cambará, Ibirarema, Ponta Grossa, Mandaguaçu, Cascavel, Luiziana and Nuporanga) of Paraná and São Paulo states (Southern Brazil), in 2002 crop season. For chemical analysis, grains of the three replications of the experiment carried out in field were mixed in same proportion to form a compost sample. The analysis in laboratory was conducted according to a complete randomized design with two replications (compost sample divided in two).

Ten grams of soybean seeds were ground in a centrifugal grinding mill, equipped with 24-tooth rotor and $0.5 \mathrm{~mm}$ stainless steel ring sieve, with the motor speed set at 15,000 rpm. This setting produced soybean flour with a uniform particle size of less than $0.25 \mathrm{~mm}$. Soluble protein was extracted for 1 hour at room temperature, while stirring a gram of full fat soybean flour in a $1: 15(\mathrm{w} / \mathrm{v})$ ratio with $0.2 \mathrm{M}$ Tris- $\mathrm{HCl}$ buffer, $\mathrm{pH} 8$, that contained $0.1 \mathrm{M}$ beta-mercaptoethanol. The mixture was centrifuged at $10,000 \mathrm{~g}$ for $10 \mathrm{~min}$ at $4^{\circ} \mathrm{C}$. After the fat layer was removed, an aliquot of $1 \mathrm{~mL}$ of the protein crude extract or supernatant was taken from each sample. Total protein concentration of each sample was determined by the method of Bradford (1976). Storage proteins and their polypeptides in the crude extract were dissociated by adding an equal volume of 5\% SDS solution and $0.1 \mathrm{M}$ beta-mercaptoethanol to each sample; then, samples were placed in boiling water bath for $10 \mathrm{~min}$ for complete dissociation. Glycerol and bromophenol blue were added to each sample to the final concentration of 10 and $0.025 \%$, respectively.

Proteins and their polypeptides were separated in a vertical slab gel apparatus, according to Chua (1980), with modifications. Each sample, containing approximately $80-100 \mu \mathrm{g}$ proteins or $10 \mu \mathrm{L}$ of protein sample, was loaded onto the gel. Proteins and polypeptides were separated using a linear gradient of 10 to $20 \%$ polyacrylamide gel. The dimension of the separating gels was $14 \times 16 \times 0.15 \mathrm{~cm}$, with 15 sample wells in stacking gel. Blank sample wells were left between loaded samples to prevent protein cross-contamination during electrophoresis and to facilitate accurate quantification by scanning densitometry after electrophoresis. Since more than one gel was required for these protein samples, and it was necessary to obtain the same polyacrylamide composition throughout the entire experiment, identical gradient gels were cast from the same polyacrylamide solution and from the same gradient-production condition. Electrophoresis of each protein sample was carried out in duplicate, at a constant current of $10 \mathrm{~mA}$ per gel at room temperature, until bromophenol blue or tracking dye reached the bottom of the gel.

Gels were stained in freshly prepared dye containing $0.25 \%$ coomassie brilliant blue $(\mathrm{w} / \mathrm{v}), 40 \%(\mathrm{v} / \mathrm{v})$ methanol and $10 \%(\mathrm{v} / \mathrm{v})$ acetic acid, and were destained in $40 \%(\mathrm{v} / \mathrm{v})$ methanol and $10 \%(\mathrm{v} / \mathrm{v})$ acetic acid. Destained gels were soaked in deionized water for at least $5 \mathrm{~min}$. Each gel was compressed and dried in a dryer. Dried gels were scanned as previously described by Kwanyuen et al. (1997), with a molecular dynamics personal densitometer equipped with a HeNe laser light source. ImageQuant software for volume integration was used in data analysis, to determine total absorbance of entire protein bands. Apparent absorbance of each protein was obtained by subtracting the background absorbance from the total absorbance of the protein bands within the same gel volume. The relative amount of each protein or polypeptide was expressed as a percentage of total protein in the same gel lane. All data were reported as means of two replications.

A completely randomized design was used to evaluate protein fraction composition of soybean, in both experiments. In the experiment 1, ANOVA and Scott \& Knott (1974) cluster analysis, at 5\% of probability, was used for grouping means of the 90 cultivars. Principal component analysis (PCA) (Johnson \& Wichern, 2007) was applied in order to have a more synthetic and informative description of the data set. The retained components consist of weighted sum of original variables in the following mathematical model: $\mathrm{PC}_{\mathrm{il}}=\mathrm{A}_{\mathrm{il}} \mathrm{X}_{1}+\ldots+\mathrm{A}_{\mathrm{in}} \mathrm{X}_{\mathrm{n}} \mathrm{i}=1,2, \ldots, \mathrm{n}$, with nine variables (alpha', alpha, beta, total 7S, acidic, basic, total 11S, $11 \mathrm{~S} / 7 \mathrm{~S}$ ratio and total fractions $=11 \mathrm{~S}+7 \mathrm{~S}$ ). The coefficients were chosen in such a way that the first component $\mathrm{PC}_{\mathrm{il}}$ had the largest variance. The output of PCA is a small number of uncorrelated components accounting for a substantial proportion of the sample data variance (Tchienkoua \& Zech, 2004). A correlation 
matrix among the variables of the protein fractions was also calculated. In the experiment 2, ANOVA and Tukey test, at $5 \%$ of probability, were used for comparing data from different sowing locations of the cultivars MG/BR 46 and IAS 5.

\section{Results and Discussion}

A significant variability for the $11 \mathrm{~S} / 7 \mathrm{~S}$ ratio was observed among the 90 Brazilian soybean cultivars, and the values ranged from 2.76 ('MS/BRS 169' -
Bacuri) to 1.17 ('BR-8' - Pelotas) (Table 1). The observed differences for protein fractions among cultivars make possible genetic manipulation to improve protein quality of soybean cultivars. Since glycinin (11S fraction) contains higher content of sulfur amino acids than beta-conglycinin (7S), it is possible to improve the amino acid balance of soybean protein. Therefore, different concentrations of protein fractions may enhance the nutritional value of soybean, as well as its properties for specific protein functionalities.

Table 1. Mean values of protein components (\%), in seeds of 90 soybean Brazilian cultivars, and group classification (GC) by Scott-Knott test ${ }^{(1)}$.

\begin{tabular}{|c|c|c|c|c|c|c|c|c|c|c|c|c|c|c|c|c|}
\hline \multirow[t]{2}{*}{ Cultivar } & \multirow[t]{2}{*}{ 11S/7S Ratio } & \multirow[t]{2}{*}{$\mathrm{GC}$} & \multicolumn{8}{|c|}{ Beta-conglycinin (7S) (\%) } & \multicolumn{6}{|c|}{ Glycinin (11S) (\%) } \\
\hline & & & $\begin{array}{l}\text { Total } \\
(7 \mathrm{~S})\end{array}$ & $\mathrm{GC}$ & alpha' & $\mathrm{GC}$ & alpha & $\mathrm{GC}$ & beta & $\mathrm{GC}$ & Acidic & $\mathrm{GC}$ & Basic & $\mathrm{GC}$ & $\begin{array}{c}\text { Total } \\
\text { (11S) }\end{array}$ & $\mathrm{GC}$ \\
\hline MS/BRS 169 (Bacuri) & 2.76 & 1 & 14.09 & 7 & 4.15 & 3 & 6.61 & 7 & 3.34 & 8 & 22.07 & 1 & 16.82 & 2 & 38.89 & 1 \\
\hline FT Cometa & 2.62 & 2 & 14.02 & 7 & 3.47 & 4 & 6.12 & 8 & 4.42 & 7 & 18.63 & 3 & 18.08 & 1 & 36.71 & 2 \\
\hline IAC 100 & 2.55 & 2 & 14.10 & 7 & 3.95 & 3 & 6.29 & 8 & 3.86 & 8 & 19.64 & 2 & 16.31 & 2 & 35.95 & 2 \\
\hline BR 38 & 2.44 & 3 & 13.56 & 7 & 3.46 & 4 & 5.54 & 9 & 4.57 & 7 & 16.93 & 5 & 16.02 & 2 & 32.95 & 3 \\
\hline IAS 5 & 2.37 & 3 & 13.11 & 7 & 2.74 & 4 & 5.41 & 9 & 4.95 & 6 & 16.24 & 5 & 14.82 & 3 & 31.06 & 3 \\
\hline MG/BR-56 (Confiança) & 2.35 & 3 & 13.39 & 7 & 3.75 & 4 & 5.45 & 9 & 4.20 & 7 & 16.48 & 5 & 14.92 & 3 & 31.41 & 3 \\
\hline IAC/PL 1 & 2.34 & 3 & 16.96 & 5 & 5.03 & 2 & 7.35 & 6 & 4.58 & 7 & 21.56 & 1 & 18.15 & 1 & 39.71 & 1 \\
\hline Embrapa 1 (IAS 5RC) & 2.32 & 3 & 14.10 & 7 & 3.14 & 4 & 5.71 & 9 & 5.25 & 6 & 16.22 & 5 & 16.43 & 2 & 32.65 & 3 \\
\hline Viçoja & 2.31 & 3 & 17.61 & 5 & 4.57 & 2 & 7.93 & 5 & 5.11 & 6 & 21.79 & 1 & 18.96 & 1 & 40.75 & 1 \\
\hline CEP 26 Umbu & 2.31 & 3 & 14.55 & 6 & 3.38 & 4 & 6.19 & 8 & 4.98 & 6 & 17.20 & 5 & 16.35 & 2 & 33.55 & 3 \\
\hline Nova IAC 7 & 2.30 & 3 & 15.49 & 6 & 4.79 & 2 & 7.36 & 6 & 3.34 & 8 & 17.95 & 4 & 17.65 & 1 & 35.61 & 2 \\
\hline IAC 20 & 2.24 & 4 & 14.17 & 7 & 3.82 & 3 & 5.16 & 9 & 5.19 & 6 & 16.40 & 5 & 15.28 & 3 & 31.68 & 3 \\
\hline Dourados & 2.21 & 4 & 18.36 & 5 & 5.30 & 1 & 8.93 & 3 & 4.13 & 7 & 21.55 & 1 & 19.01 & 1 & 40.56 & 1 \\
\hline Mineira & 2.18 & 4 & 17.42 & 5 & 5.12 & 1 & 7.41 & 6 & 4.89 & 7 & 20.68 & 2 & 17.23 & 1 & 37.91 & 1 \\
\hline J 200 & 2.17 & 4 & 17.35 & 5 & 4.82 & 2 & 8.03 & 4 & 4.50 & 7 & 20.61 & 2 & 17.08 & 1 & 37.69 & 1 \\
\hline DM Rainha & 2.17 & 4 & 17.22 & 5 & 4.49 & 2 & 7.71 & 5 & 5.02 & 6 & 18.69 & 3 & 18.62 & 1 & 37.32 & 1 \\
\hline FT Líder & 2.16 & 4 & 13.63 & 7 & 3.63 & 4 & 5.43 & 9 & 4.57 & 7 & 14.85 & 7 & 14.50 & 3 & 29.34 & 4 \\
\hline EMGOPA 310 & 2.12 & 4 & 16.88 & 5 & 4.82 & 2 & 7.00 & 6 & 5.06 & 6 & 18.11 & 4 & 17.70 & 1 & 35.81 & 2 \\
\hline UFV 15 (Uberlândia) & 2.12 & 4 & 16.53 & 5 & 4.55 & 2 & 7.22 & 6 & 4.77 & 7 & 18.36 & 4 & 16.70 & 2 & 35.06 & 2 \\
\hline Embrapa 19 & 2.11 & 4 & 15.64 & 6 & 3.56 & 4 & 6.18 & 8 & 5.91 & 5 & 17.66 & 4 & 15.31 & 3 & 32.97 & 3 \\
\hline MG/BR 54 (Renascença) & 2.10 & 4 & 17.04 & 5 & 4.76 & 2 & 7.56 & 5 & 4.72 & 7 & 19.20 & 3 & 16.68 & 2 & 35.87 & 2 \\
\hline Embrapa 25 & 2.08 & 5 & 19.18 & 4 & 4.79 & 2 & 8.57 & 4 & 5.83 & 5 & 21.57 & 1 & 18.40 & 1 & 39.97 & 1 \\
\hline FT 19 (Macachá) & 2.04 & 5 & 17.91 & 5 & 4.94 & 2 & 7.07 & 6 & 5.90 & 5 & 19.39 & 3 & 17.17 & 1 & 36.56 & 2 \\
\hline MG/BR 46 (Conquista) & 2.04 & 5 & 17.48 & 5 & 5.44 & 1 & 7.01 & 6 & 5.03 & 6 & 18.96 & 3 & 16.68 & 2 & 35.64 & 2 \\
\hline Santa Maria & 2.03 & 5 & 17.46 & 5 & 4.98 & 2 & 6.59 & 7 & 5.89 & 5 & 18.20 & 4 & 17.19 & 1 & 35.39 & 2 \\
\hline IAS 4 & 2.02 & 5 & 14.90 & 6 & 3.37 & 4 & 5.79 & 9 & 5.74 & 5 & 16.34 & 5 & 13.80 & 4 & 30.13 & 4 \\
\hline BRS 155 & 2.01 & 5 & 16.61 & 5 & 5.33 & 1 & 6.98 & 6 & 4.31 & 7 & 17.83 & 4 & 15.55 & 2 & 33.38 & 3 \\
\hline São Luiz & 1.97 & 6 & 18.59 & 4 & 4.51 & 2 & 7.66 & 5 & 6.42 & 4 & 19.27 & 3 & 17.28 & 1 & 36.55 & 2 \\
\hline IAC 2 & 1.96 & 6 & 17.92 & 5 & 4.75 & 2 & 7.05 & 6 & 6.12 & 5 & 19.00 & 3 & 16.23 & 2 & 35.24 & 2 \\
\hline FT 2 & 1.96 & 6 & 20.50 & 3 & 4.53 & 2 & 9.40 & 3 & 6.57 & 4 & 21.06 & 1 & 19.16 & 1 & 40.22 & 1 \\
\hline MS/BRS 168 (Piapara) & 1.95 & 6 & 19.45 & 4 & 5.34 & 1 & 7.37 & 6 & 6.74 & 4 & 20.13 & 2 & 17.74 & 1 & 37.87 & 1 \\
\hline OCEPAR 9 SS1 & 1.94 & 6 & 19.02 & 4 & 4.71 & 2 & 8.29 & 4 & 6.01 & 5 & 19.04 & 3 & 17.89 & 1 & 36.93 & 2 \\
\hline FT 9 (Inaê) & 1.91 & 6 & 16.16 & 5 & 3.37 & 4 & 5.92 & 8 & 6.88 & 4 & 17.13 & 5 & 13.79 & 4 & 30.92 & 3 \\
\hline OCEPAR 14 & 1.91 & 6 & 17.38 & 5 & 3.55 & 4 & 7.07 & 6 & 6.75 & 4 & 18.04 & 4 & 15.11 & 3 & 33.16 & 3 \\
\hline BRS 158 (Milena) & 1.90 & 6 & 16.58 & 5 & 4.19 & 3 & 7.26 & 6 & 5.14 & 6 & 16.96 & 5 & 14.58 & 3 & 31.54 & 3 \\
\hline IPB-T & 1.90 & 6 & 17.30 & 5 & 4.56 & 2 & 7.21 & 6 & 5.53 & 5 & 17.53 & 5 & 15.35 & 3 & 32.88 & 3 \\
\hline OCEPAR 19 (Cotia) & 1.88 & 6 & 16.52 & 5 & 3.58 & 4 & 5.86 & 9 & 7.09 & 3 & 15.39 & 6 & 15.71 & 2 & 31.10 & 3 \\
\hline União & 1.88 & 6 & 16.83 & 5 & 4.17 & 3 & 6.91 & 7 & 5.75 & 5 & 16.41 & 5 & 15.25 & 3 & 31.65 & 3 \\
\hline SPS 1 & 1.87 & 6 & 20.58 & 3 & 4.34 & 3 & 8.65 & 4 & 7.59 & 2 & 19.95 & 2 & 18.45 & 1 & 38.40 & 1 \\
\hline IAS 3 (Delta) & 1.86 & 6 & 18.73 & 4 & 4.31 & 3 & 8.13 & 4 & 6.30 & 4 & 18.78 & 3 & 15.97 & 2 & 34.75 & 2 \\
\hline OCEPAR 2 (IAPÓ) & 1.85 & 6 & 15.26 & 6 & 3.12 & 4 & 6.31 & 8 & 5.83 & 5 & 15.43 & 6 & 12.80 & 4 & 28.23 & 4 \\
\hline MG/BR 22 (Garimpo) & 1.85 & 7 & 18.60 & 4 & 5.41 & 1 & 8.92 & 3 & 4.27 & 7 & 18.40 & 4 & 15.94 & 2 & 34.34 & 2 \\
\hline BRS 133 & 1.84 & 7 & 20.68 & 3 & 5.21 & 1 & 8.81 & 3 & 6.66 & 4 & 20.42 & 2 & 17.74 & 1 & 38.16 & 1 \\
\hline BRS 132 & 1.84 & 7 & 13.92 & 7 & 3.52 & 4 & 6.21 & 8 & 4.20 & 7 & 13.67 & 7 & 11.91 & 4 & 25.58 & 4 \\
\hline
\end{tabular}


In soybean breeding programs, whose main objective is to obtain high quality protein, the $11 \mathrm{~S} / 7 \mathrm{~S}$ ratio has been a criterion of indirect selection for this trait. Through the analysis of $11 \mathrm{~S} / 7 \mathrm{~S}$ ratio data, it is possible to select high or low $11 \mathrm{~S}$ or $7 \mathrm{~S}$ lines or cultivars. Cultivar MS/BRS 169 (Bacuri) presented $11 \mathrm{~S} / 7 \mathrm{~S}$ ratio of 2.76 , which is relevant, when compared with observations of Kitamura \& Kaizuma (1981), who reported $11 \mathrm{~S} / 7 \mathrm{~S}$ ratio of 2.59 for the genotype Mo-shi-dou, which is the germplasm source for high levels of the $11 \mathrm{~S}$ protein fractions. The same authors observed a ratio of $11 \mathrm{~S} / 7 \mathrm{~S}$ of 1.12 for normal strains. Therefore, 'MS/BRS 169'(Bacuri) could be a genetic source to increase glycinin content for Brazilian soybean breeding programs.

'MS-BRS 169' (Bacuri) and 'BR-8' (Pelotas) presented the highest and the lowest $11 \mathrm{~S} / 7 \mathrm{~S}$ ratios $(2.76$ and 1.17, respectively), among all the 90 genotypes. Content of beta-conglycinin (7S) protein subfractions alpha', alpha, and beta were, respectively, 4.1, 6.6, and

Table 1. Continuation

\begin{tabular}{|c|c|c|c|c|c|c|c|c|c|c|c|c|c|c|c|c|}
\hline \multirow[t]{2}{*}{ Cultivar } & \multirow[t]{2}{*}{ 11S/7S Ratio } & \multirow[t]{2}{*}{$\mathrm{GC}$} & \multicolumn{8}{|c|}{ Beta-conglycinin (7S) $(\%)$} & \multicolumn{6}{|c|}{ Glycinin (11S) (\%) } \\
\hline & & & $\begin{array}{l}\text { Total } \\
(7 \mathrm{~S})\end{array}$ & $\mathrm{GC}$ & alpha' & $\mathrm{GC}$ & alpha & $\mathrm{GC}$ & beta & $\mathrm{GC}$ & Acidic & $\mathrm{GC}$ & Basic & $\mathrm{GC}$ & $\begin{array}{l}\text { Total } \\
(11 \mathrm{~S}) \\
\end{array}$ & $\mathrm{GC}$ \\
\hline Santa Rosa & 1.84 & 7 & 20.37 & 3 & 5.53 & 1 & 7.84 & 5 & 7.01 & 3 & 20.02 & 2 & 17.42 & 1 & 37.44 & 1 \\
\hline DM Soberana & 1.83 & 7 & 20.46 & 3 & 5.02 & 2 & 8.30 & 4 & 7.14 & 3 & 20.50 & 2 & 17.06 & 1 & 37.56 & 1 \\
\hline Embrapa 26 & 1.83 & 7 & 19.16 & 4 & 4.39 & 2 & 7.83 & 5 & 6.94 & 3 & 17.87 & 4 & 17.20 & 1 & 35.07 & 2 \\
\hline BR 6 (Nova Bragg) & 1.81 & 7 & 20.77 & 3 & 4.97 & 2 & 8.05 & 4 & 7.75 & 2 & 20.18 & 2 & 17.47 & 1 & 37.66 & 1 \\
\hline BR 14 (Modelo) & 1.81 & 7 & 18.78 & 4 & 5.12 & 1 & 7.26 & 6 & 6.40 & 4 & 18.92 & 3 & 15.07 & 3 & 33.99 & 2 \\
\hline BRS 138 & 1.80 & 7 & 18.15 & 5 & 4.35 & 3 & 8.12 & 4 & 5.68 & 5 & 16.46 & 5 & 16.28 & 2 & 32.74 & 3 \\
\hline RS 5 (Esmeralda) & 1.79 & 7 & 20.00 & 3 & 5.85 & 1 & 8.18 & 4 & 5.96 & 5 & 18.96 & 3 & 16.92 & 2 & 35.88 & 2 \\
\hline CEP 10 & 1.79 & 7 & 15.93 & 5 & 3.44 & 4 & 6.53 & 7 & 5.95 & 5 & 15.54 & 6 & 13.03 & 4 & 28.56 & 4 \\
\hline Embrapa 48 & 1.79 & 7 & 15.23 & 6 & 3.64 & 4 & 5.84 & 9 & 5.75 & 5 & 13.86 & 7 & 13.41 & 4 & 27.26 & 4 \\
\hline IAC Holambra Stwart 1 & 1.78 & 7 & 21.22 & 3 & 5.00 & 2 & 7.98 & 5 & 8.25 & 2 & 20.48 & 2 & 17.37 & 1 & 37.85 & 1 \\
\hline EMGOPA 311 & 1.78 & 7 & 19.15 & 4 & 5.14 & 1 & 8.16 & 4 & 5.85 & 5 & 18.30 & 4 & 15.83 & 2 & 34.14 & 2 \\
\hline BRS 153 & 1.78 & 7 & 17.96 & 5 & 3.97 & 3 & 7.74 & 5 & 6.25 & 4 & 15.74 & 6 & 16.14 & 2 & 31.89 & 3 \\
\hline Davis & 1.78 & 7 & 16.43 & 5 & 3.30 & 4 & 6.34 & 8 & 6.80 & 4 & 15.93 & 5 & 13.26 & 4 & 29.19 & 4 \\
\hline OCEPAR 15 (Paracatu) & 1.78 & 7 & 20.79 & 3 & 5.37 & 1 & 8.88 & 3 & 6.53 & 4 & 19.29 & 3 & 17.63 & 1 & 36.92 & 2 \\
\hline BR 4 & 1.77 & 7 & 16.54 & 5 & 3.32 & 4 & 6.07 & 8 & 7.15 & 3 & 15.64 & 6 & 13.60 & 4 & 29.24 & 4 \\
\hline CD 202 & 1.76 & 7 & 15.24 & 6 & 3.50 & 4 & 6.13 & 8 & 5.61 & 5 & 14.90 & 7 & 11.97 & 4 & 26.87 & 4 \\
\hline BRS 135 & 1.76 & 7 & 20.75 & 3 & 4.98 & 2 & 7.89 & 5 & 7.88 & 2 & 19.37 & 3 & 17.22 & 1 & 36.59 & 2 \\
\hline Campos Gerais & 1.73 & 7 & 17.46 & 5 & 4.12 & 3 & 6.42 & 8 & 6.92 & 3 & 15.43 & 6 & 14.69 & 3 & 30.12 & 4 \\
\hline MG/BR 58 (Segurança) & 1.71 & 7 & 20.62 & 3 & 4.60 & 2 & 7.93 & 5 & 8.09 & 2 & 18.31 & 4 & 16.96 & 2 & 35.27 & 2 \\
\hline Bienville & 1.71 & 7 & 21.25 & 3 & 5.02 & 2 & 8.84 & 3 & 7.39 & 3 & 19.68 & 2 & 16.61 & 2 & 36.29 & 2 \\
\hline FT 6 (Veneza) & 1.69 & 8 & 17.94 & 5 & 3.32 & 4 & 6.63 & 7 & 7.98 & 2 & 17.54 & 5 & 12.72 & 4 & 30.27 & 4 \\
\hline MS/BR20 (Ipê) & 1.68 & 8 & 21.46 & 3 & 5.14 & 1 & 9.06 & 3 & 7.26 & 3 & 19.82 & 2 & 16.25 & 2 & 36.07 & 2 \\
\hline MG/BR 66 (Liderança) & 1.68 & 8 & 20.85 & 3 & 5.26 & 1 & 7.55 & 5 & 8.04 & 2 & 18.70 & 3 & 16.35 & 2 & 35.05 & 2 \\
\hline MS/BRS 170 (Taquari) & 1.65 & 8 & 20.79 & 3 & 4.29 & 3 & 8.28 & 4 & 8.21 & 2 & 18.22 & 4 & 16.09 & 2 & 34.31 & 2 \\
\hline BRS 134 & 1.65 & 8 & 19.77 & 4 & 4.21 & 3 & 8.06 & 4 & 7.50 & 3 & 18.04 & 4 & 14.52 & 3 & 32.57 & 3 \\
\hline IAC 1 & 1.64 & 8 & 21.32 & 3 & 4.93 & 2 & 9.86 & 2 & 6.53 & 4 & 19.18 & 3 & 15.91 & 2 & 35.09 & 2 \\
\hline Ivaí & 1.64 & 8 & 20.71 & 3 & 5.02 & 2 & 8.38 & 4 & 7.32 & 3 & 18.82 & 3 & 15.07 & 3 & 33.89 & 2 \\
\hline Ivorá & 1.63 & 8 & 17.83 & 5 & 3.69 & 4 & 6.89 & 7 & 7.25 & 3 & 15.37 & 6 & 13.73 & 4 & 29.10 & 4 \\
\hline OCEPAR 6 & 1.62 & 8 & 18.63 & 4 & 4.27 & 3 & 7.74 & 5 & 6.62 & 4 & 16.98 & 5 & 13.19 & 4 & 30.17 & 4 \\
\hline IAC 16 & 1.62 & 8 & 18.20 & 5 & 3.80 & 3 & 6.63 & 7 & 7.78 & 2 & 14.93 & 7 & 14.54 & 3 & 29.47 & 4 \\
\hline BRS 156 & 1.62 & 8 & 17.90 & 5 & 4.01 & 3 & 7.32 & 6 & 6.58 & 4 & 15.77 & 6 & 13.17 & 4 & 28.94 & 4 \\
\hline KI-S 702 & 1.60 & 8 & 21.03 & 3 & 4.30 & 3 & 9.31 & 3 & 7.42 & 3 & 17.80 & 4 & 15.59 & 2 & 33.39 & 3 \\
\hline Cobb & 1.59 & 8 & 21.25 & 3 & 4.11 & 3 & 7.63 & 5 & 9.51 & 1 & 17.90 & 4 & 15.93 & 2 & 33.83 & 2 \\
\hline Vila Rica & 1.59 & 8 & 23.00 & 2 & 6.07 & 1 & 9.38 & 3 & 7.55 & 2 & 20.06 & 2 & 16.34 & 2 & 36.40 & 2 \\
\hline Embrapa 4 (BR 4 RC) & 1.58 & 8 & 20.60 & 3 & 4.55 & 2 & 8.31 & 4 & 7.75 & 2 & 17.23 & 5 & 15.41 & 3 & 32.64 & 3 \\
\hline FT Jatobá & 1.54 & 8 & 23.20 & 2 & 5.11 & 1 & 10.38 & 1 & 7.71 & 2 & 19.83 & 2 & 15.90 & 2 & 35.73 & 2 \\
\hline BRS 137 & 1.53 & 8 & 18.63 & 4 & 4.57 & 2 & 6.77 & 7 & 7.29 & 3 & 14.74 & 7 & 13.77 & 4 & 28.51 & 4 \\
\hline BR 23 & 1.52 & 8 & 21.76 & 2 & 4.71 & 2 & 9.16 & 3 & 7.88 & 2 & 18.12 & 4 & 14.95 & 3 & 33.06 & 3 \\
\hline BRS 62 & 1.50 & 9 & 25.42 & 1 & 5.52 & 1 & 10.73 & 1 & 9.18 & 1 & 20.42 & 2 & 17.78 & 1 & 38.20 & 1 \\
\hline OCEPAR 16 & 1.47 & 9 & 20.23 & 3 & 4.79 & 2 & 8.87 & 3 & 6.58 & 4 & 15.76 & 6 & 14.02 & 4 & 29.78 & 4 \\
\hline FT 14 (Piracema) & 1.46 & 9 & 20.17 & 3 & 4.14 & 3 & 9.02 & 3 & 7.00 & 3 & 16.14 & 5 & 13.36 & 4 & 29.49 & 4 \\
\hline BR 36 & 1.46 & 9 & 23.39 & 2 & 4.98 & 2 & 9.28 & 3 & 9.12 & 1 & 18.45 & 4 & 15.71 & 2 & 34.15 & 2 \\
\hline FT 4 & 1.44 & 9 & 20.55 & 3 & 4.68 & 2 & 8.41 & 4 & 7.47 & 3 & 16.35 & 5 & 13.26 & 4 & 29.61 & 4 \\
\hline BR 12 & 1.44 & 9 & 21.36 & 3 & 4.76 & 2 & 9.20 & 3 & 7.40 & 3 & 16.08 & 5 & 14.62 & 3 & 30.70 & 3 \\
\hline BR 16 & 1.36 & 9 & 22.17 & 2 & 4.52 & 2 & 9.22 & 3 & 8.43 & 2 & 15.93 & 5 & 14.26 & 3 & 30.19 & 4 \\
\hline BR 8 (Pelotas) & 1.18 & 9 & 23.35 & 2 & 5.52 & 1 & 10.15 & 2 & 7.69 & 2 & 14.52 & 7 & 12.92 & 4 & 27.43 & 4 \\
\hline
\end{tabular}

${ }^{(1)}$ Groups determined by Scott-Knott cluster analysis, at $5 \%$ of probability. 
$3.3 \%$ for the cultivar MS/BRS 169 (Bacuri), and 5.5, 10.1, and $7.7 \%$ for 'BR-8' (Pelotas). Glycinin (11S) acidic and basic subfractions were, respectively, 22.1 and $16.8 \%$ for cultivar MS/BRS 169 , and 14.5 and $12.9 \%$ for cultivar BR-8 (Pelotas) (Table 1). Fehr et al. (2003) found $11 \mathrm{~S} / 7 \mathrm{~S}$ ratio of 2.04 for cultivar Vinton 81 and 1.51 for cultivar IA2021, in a study of three years in eight locations. 'Vinton 81 ' has good performance for tofu processing, which may be due to its high content of glycinin (11S). Because of its high 11S/7S ratio, the cultivar MS/BRS 169 (Bacuri) may have the same properties.

Strong positive correlations were observed among total protein frations [Glycinin (11S) and beta-conglycinin $(7 \mathrm{~S})$ ] with total $11 \mathrm{~S}$, and total $7 \mathrm{~S}$ (Table 2). For $11 \mathrm{~S} / 7 \mathrm{~S}$ ratio, inverse significant correlations were observed for beta-conglycinin (7S) subunits (alpha and beta) and for total $7 \mathrm{~S}$ fraction, as expected. A positive significant correlation, although weak, was observed for total $11 \mathrm{~S}$ protein fraction and $11 \mathrm{~S} / 7 \mathrm{~S}$ ratio. Protein content exhibited no significant correlation with other protein components, as already observed by Harada et al. (1983) and Fehr et al. (2003). These data confirm the possibility of breeding for individual protein fractions and their subunits without affecting protein content. Yaklich et al. (2001), who studied the contribution of the two major protein fractions, in high seed protein lines of soybean, found higher concentration of these compounds in those lines than in normal-protein soybean lines.

Among the 90 cultivars sown in the same location, it was observed that beta-conglycinin (7S) protein fractions presented more variability than glycinin (11S) protein fractions. The
Scott-Knott grouping test classified $7 \mathrm{~S}$ proteins in 7 groups, $11 \mathrm{~S}$ proteins in 4 groups, and protein fraction ratios (11S/7S) in 9 groups (Table 1). For the highest content of glycinin (11S), classified at group 1 by Scott-Knott test, it was observed a range of $40.75 \%$ (cultivar Viçoja) to $37.32 \%$ (DM Rainha). However, within the groups 1, 2 and 3, which presented the highest values for $11 \mathrm{~S} / 7 \mathrm{~S}$ ratios, just cultivars MS/BRS 169 (Bacuri), IAC/ PL 1 and Viçoja presented high values for glycinin content, and were in group 1 (Table 1).

Inverse relationship between total $7 \mathrm{~S}$ protein fractions and protein ratios can be observed at the Scott-Knott grouping test, where the majority of the cultivars that presented low content of beta-conglycinin (7S) (groups 6 and 7) - which included cultivars IAC 20, FT-Líder, EMBRAPA 19, IAS 4, OCEPAR 2 (Iapó), BRS 132, EMBRAPA 48 and CD 202 - also presented low content of 7S protein fractions (Table 1), but were not in the first groups for the $11 \mathrm{~S} / 7 \mathrm{~S}$ ratios. Cultivar BRS 132, in group 7 , for both total $7 \mathrm{~S}$ and $11 \mathrm{~S} / 7 \mathrm{~S}$ ratio $(13.92 \%$ and 1.84 , respectively) because of the low content of $11 \mathrm{~S}$ protein fraction $(25.58 \%)$, was classified in group 4 for this fraction (Table 1).

Cultivars that presented the lowest $11 \mathrm{~S} / 7 \mathrm{~S}$ ratios (group 9) also presented the highest values for total beta-conglycinin (7S), except for 'BRS 62', that is in group 1 for total $7 \mathrm{~S}$ and $11 \mathrm{~S}$ (Table 1). Cultivar BRS 62 presented the highest content of beta-conglycinin (7S) $(25.4 \%)$, while cultivar IAS 5 had the lowest (13.11\%); these cultivars had $11 \mathrm{~S} / 7 \mathrm{~S}$ ratios of 1.50 and 2.37 , respectively.

Figure 1 shows the functional relationship and closeness among the variables (protein fractions components). This two-dimensional representation

Table 2. Correlations among storage protein fractions in soybean seeds of 90 Brazilian cultivars ${ }^{(1)}$.

\begin{tabular}{|c|c|c|c|c|c|c|c|c|c|c|}
\hline Protein fractions & Alpha' & Alpha & Beta & Total 7S & Acidic & Basic & Total 11S & $\begin{array}{c}\text { Ratio } \\
11 \mathrm{~S} / 7 \mathrm{~S}\end{array}$ & $\begin{array}{c}\text { Total } \\
7 \mathrm{~S}+11 \mathrm{~S}\end{array}$ & $\begin{array}{c}\text { Protein } \\
(\%)\end{array}$ \\
\hline Alpha' & 1.00 & & & & & & & & & \\
\hline Alpha & $0.71 * * *$ & 1.00 & & & & & & & & \\
\hline Beta & 0.18 & $0.49 * * *$ & 1.00 & & & & & & & \\
\hline Total 7S & $0.69 * * *$ & $0.90 * * *$ & $0.78 * * *$ & 1.00 & & & & & & \\
\hline Acidic & $0.60 * * *$ & $0.44 * * *$ & -0.04 & 0.34 & 1.00 & & & & & \\
\hline Basic & $0.51 * * *$ & 0.30 & -0.15 & 0.20 & $0.82 * * *$ & 1.00 & & & & \\
\hline Total 11S & $0.58 * * *$ & $0.39 * * *$ & -0.10 & 0.29 & $0.96 * * *$ & $0.95 * * *$ & 1.00 & & & \\
\hline Ratio $11 \mathrm{~S} / 7 \mathrm{~S}$ & -0.27 & $-0.58 * * *$ & $-0.81 * * *$ & $-0.75 * * *$ & 0.31 & $0.44 * * *$ & $0.39 * * *$ & 1.00 & & \\
\hline Total $7 \mathrm{~S}+11 \mathrm{~S}$ & $0.78 * * *$ & $0.76^{* * *}$ & $0.35 * * *$ & $0.74 * * *$ & $0.86 * * *$ & $0.77 * * *$ & $0.86^{* * *} *$ & -0.13 & 1.00 & \\
\hline Protein $(\%)$ & 0.02 & -0.06 & -0.16 & -0.10 & 0.21 & 0.08 & 0.16 & 0.21 & 0.05 & 1.00 \\
\hline
\end{tabular}

***Significant at $0,1 \%$ of probability; $\mathrm{N}=90$. 
of the whole data set displays $89.5 \%$ of the total protein fractions variance (TPFV) and provides a grouping of variables, according to their relative position in the plane defined by principal component analysis 1 (PC1) and principal component analysis 2 (PC2). The first component PC1 (horizontal axis), associated with $55.4 \%$ of the TPFV, displays strong and negative loadings on almost all the protein fractions, except for the subunit beta, which is more negative in relation

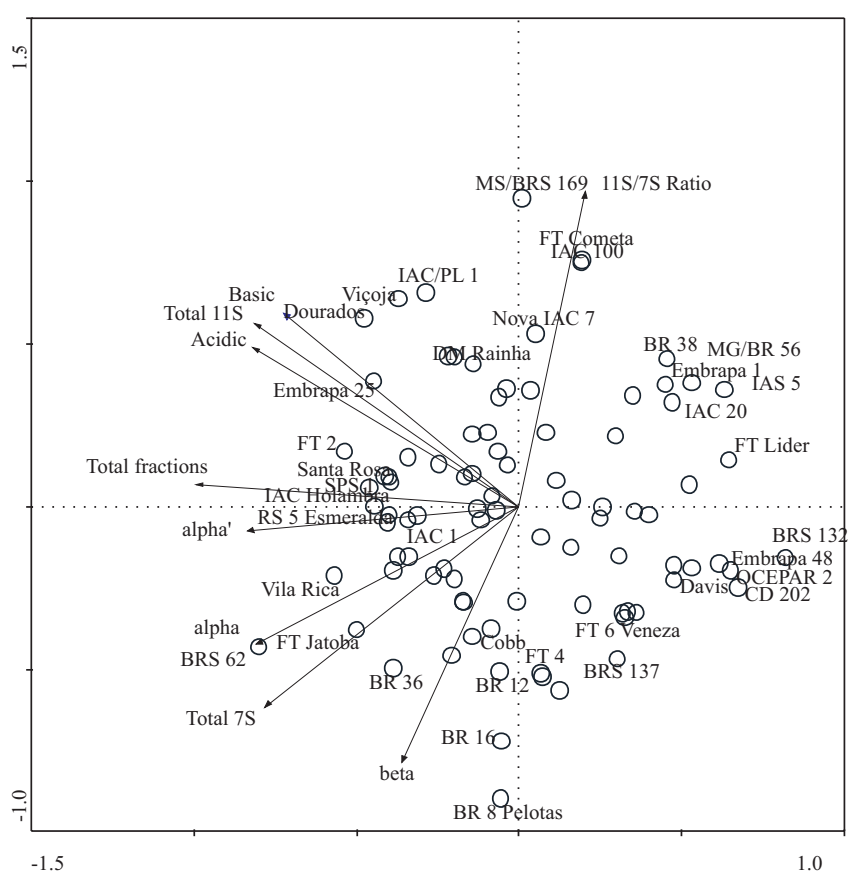

Figure 1. Principal component analysis of protein fractions and $11 \mathrm{~S} / 7 \mathrm{~S}$ ratios, of 90 Brazilian cultivars, displaying the 5 highest and the 5 smallest of each fraction. to the second component PC2 (vertical axis) and the $11 \mathrm{~S} / 7 \mathrm{~S}$ ratio, that is strongly positive related to second component either. Cultivars whose projections are at the end of the arrows (on crescent direction) are those that had high concentration of that protein fraction component, and, inversely, those that have extreme projections, at the decrescent direction, had less concentration of the specific protein component. Therefore, cultivar MS/BRS 169 (Bacuri) and cultivar BR-8 (Pelotas), which had the extreme values for $11 \mathrm{~S} / 7 \mathrm{~S}$ ratios, are located in inverse extreme positions in the Figure 1, according to data in Table 1.

In experiment 2, when cultivar IAS 5 was sown in 12 different locations of Paraná and São Paulo states, Southern Brazil $\left(23^{\circ} \mathrm{S}\right)$, it was observed a large variability for total glycinin (11S) and beta-conglycinin $(7 \mathrm{~S})$ protein fractions. For total glycinin, this variability ranged from $29.6 \%$, in Nuporanga, to $39.8 \%$, in Pirassununga. Variability for total beta-conglycinin protein fraction ranged from $13.6 \%$, in Londrina, to $18.6 \%$, in Nuporanga (Table 3). Inverse relationship between $11 \mathrm{~S}$ and $7 \mathrm{~S}$ fractions can be observed in Nuporanga, where the highest and the lowest concentrations occurred for both protein fractions (7S and 11S) (Table 3). In this location, it was also observed the lowest value of 1.59 for the $11 \mathrm{~S} / 7 \mathrm{~S}$ ratio. Cascavel was the location that presented high content of $7 \mathrm{~S}$ and $11 \mathrm{~S}$ protein fractions, while Londrina presented the lowest $7 \mathrm{~S}$ concentration (Table 3). By the data of cultivar IAS 5 , for $11 \mathrm{~S}$ and $7 \mathrm{~S}$ protein fractions concentrations and $11 \mathrm{~S} / 7 \mathrm{~S}$ ratio, it can be observed that sowing location

Table 3. Glycinin and beta-conglycinin protein fraction composition (\%), in seeds of soybean cultivar IAS 5, sown in 12 different locations of Paraná and São Paulo states ${ }^{(1)}$.

\begin{tabular}{|c|c|c|c|c|c|c|c|c|c|}
\hline \multirow[t]{2}{*}{ Locations } & \multicolumn{4}{|c|}{ Beta-Conglycinin (7S) } & \multicolumn{3}{|c|}{ Glycinin (11S) } & \multirow{2}{*}{$\begin{array}{r}11 \mathrm{~S} / 7 \mathrm{~S} \\
\text { Ratio }\end{array}$} & \multirow{2}{*}{$\begin{array}{c}\text { Total } \\
7 \mathrm{~S}+11 \mathrm{~S}\end{array}$} \\
\hline & Alpha' & Alpha & Beta & Total 7S & Acidic & Basic & Total 11S & & \\
\hline Nuporanga & $3.98 \mathrm{a}$ & $6.64 \mathrm{abc}$ & $7.93 \mathrm{a}$ & $18.56 \mathrm{a}$ & $15.34 \mathrm{~d}$ & $14.27 \mathrm{~b}$ & $29.61 c$ & $1.59 \mathrm{~d}$ & $48.17 \mathrm{ab}$ \\
\hline Cascavel & $4.93 \mathrm{a}$ & $7.27 \mathrm{a}$ & $5.95 b$ & $18.16 \mathrm{ab}$ & $19.23 \mathrm{ab}$ & $17.86 \mathrm{ab}$ & $37.09 \mathrm{ab}$ & $2.04 \mathrm{bc}$ & $55.25 \mathrm{ab}$ \\
\hline Mandaguaçu & $4.73 a$ & $7.03 \mathrm{ab}$ & $5.80 \mathrm{~b}$ & $17.58 \mathrm{abc}$ & $18.67 \mathrm{abc}$ & $18.36 \mathrm{ab}$ & $37.03 \mathrm{ab}$ & $2.10 \mathrm{abc}$ & $54.61 \mathrm{ab}$ \\
\hline Luiziana & $4.45 \mathrm{a}$ & $7.01 \mathrm{ab}$ & $5.69 \mathrm{bc}$ & $17.16 \mathrm{abc}$ & $16.84 \mathrm{bcd}$ & $15.91 \mathrm{ab}$ & $32.75 \mathrm{abc}$ & $1.90 \mathrm{c}$ & $49.90 \mathrm{ab}$ \\
\hline Pirassununga & $4.44 a$ & $6.65 \mathrm{abc}$ & $5.99 b$ & $17.09 \mathrm{abc}$ & $20.39 a$ & $19.38 \mathrm{a}$ & $39.78 \mathrm{a}$ & $2.33 \mathrm{a}$ & $56.86 \mathrm{a}$ \\
\hline Ponta Grossa & $4.37 \mathrm{a}$ & $6.39 \mathrm{abc}$ & $5.25 \mathrm{bcd}$ & $16.02 \mathrm{abc}$ & 17.90abcd & $16.70 \mathrm{ab}$ & $34.60 \mathrm{abc}$ & $2.16 \mathrm{abc}$ & $50.62 \mathrm{ab}$ \\
\hline Guaíra & $4.00 \mathrm{a}$ & $6.21 \mathrm{abc}$ & $4.73 \mathrm{bcd}$ & $14.94 \mathrm{abc}$ & $18.27 \mathrm{abc}$ & $17.04 \mathrm{ab}$ & $35.31 \mathrm{abc}$ & $2.36 \mathrm{a}$ & $50.25 \mathrm{ab}$ \\
\hline Ibirarema & $4.19 a$ & $6.00 \mathrm{abc}$ & $4.58 \mathrm{def}$ & $14.77 \mathrm{abc}$ & $16.40 \mathrm{bcd}$ & $15.67 \mathrm{ab}$ & $32.07 \mathrm{bc}$ & $2.17 \mathrm{abc}$ & $46.84 \mathrm{ab}$ \\
\hline Morro Agudo & $3.54 \mathrm{a}$ & $5.70 \mathrm{c}$ & $5.36 \mathrm{bcd}$ & $14.61 \mathrm{abc}$ & $17.30 \mathrm{bcd}$ & $15.96 \mathrm{ab}$ & $33.25 \mathrm{abc}$ & $2.27 \mathrm{ab}$ & $47.86 \mathrm{ab}$ \\
\hline Cambará & $4.08 \mathrm{a}$ & $6.02 \mathrm{abc}$ & $4.16 \mathrm{ef}$ & $14.27 \mathrm{bc}$ & $17.02 \mathrm{bcd}$ & $15.04 \mathrm{ab}$ & $32.07 \mathrm{bc}$ & $2.24 \mathrm{ab}$ & $46.34 \mathrm{ab}$ \\
\hline Pedrinhas & $3.69 \mathrm{a}$ & $5.55 \mathrm{c}$ & $4.43 \mathrm{def}$ & $13.69 \mathrm{c}$ & $16.80 \mathrm{bcd}$ & $15.34 \mathrm{ab}$ & $32.15 b c$ & $2.35 \mathrm{a}$ & $45.83 b$ \\
\hline Londrina & $4.05 \mathrm{a}$ & $5.92 b c$ & $3.59 \mathrm{f}$ & $13.58 \mathrm{c}$ & $16.29 \mathrm{~cd}$ & $15.02 \mathrm{ab}$ & $31.31 b c$ & $2.30 \mathrm{ab}$ & $44.89 b$ \\
\hline
\end{tabular}

${ }^{(1)}$ Means followed by the same letters, in the columns, do not differ by Tukey test, at $5 \%$ of probability. 
affected concentrations of these compounds. High differences for $11 \mathrm{~S}$ fractions were also observed in Cascavel, Mandaguaçu, Ponta Grossa and Guaíra. In 12 locations in the Southern region of Brazil, the range for $11 \mathrm{~S} / 7 \mathrm{~S}$ ratio was 2.36 to 1.59 , which was similar to findings of Fehr et al. (2003), who reported differences among year-location combinations.

Data of individual beta-conglycinin subunits (Table 3 ) confirm the inverse relationship of $11 \mathrm{~S} / 7 \mathrm{~S}$ ratio and total $7 \mathrm{~S}$ protein fraction. Nuporanga presented the highest values for beta subunit and total beta-conglycinin $(7 \mathrm{~S})$, while Londrina presented the smallest concentrations for the same subunits. In Pirassununga, the cultivar IAS 5 presented the highest concentration of the acidic and basic subunits of glycinin. Larger concentrations of individual subunits for both beta-conglycinin (7S) and glycinin (11S) were observed in Cascavel (Table 3 ), but the $11 \mathrm{~S} / 7 \mathrm{~S}$ ratio was smaller (2.04), as compared to other locations. Londrina presented low concentrations of acidic and basic subunits of glycinin, and the lowest value for beta-conglycinin subunits, although the $11 \mathrm{~S} / 7 \mathrm{~S}$ ratio was not the smallest (2.30). Londrina presented low precipitation $(121 \mathrm{~mm})$ during the filling period months (February, March and April) in 2002, while Nuporanga had good water availability $(455 \mathrm{~mm})$ during the same period (www.agritempo.org.br - September 2006). Observing data of Londrina and Nuporanga, it appears that beta-conglycinin protein fractions may be more susceptible to water stress than glycinin fractions.

Larger variability for beta-conglycinin than for glycinin protein fractions was observed, when cultivar MG/BRS 46 Conquista was sown in 16 locations of Central Brazil $\left(17^{\circ} \mathrm{S}\right)$ (Table 4). Among these locations, total 7S protein fractions ranged from $16.3 \%$, in Rio Verde, to $24.9 \%$ in Cristalina-1, and $11 \mathrm{~S} / 7 \mathrm{~S}$ ratio was 1.89 and 1.96 , respectively. Total $11 \mathrm{~S}$ protein fractions ranged from $26.4 \%$, in Chapadão do Céu, to $39.4 \%$, in Senador Canedo, while $11 \mathrm{~S} / 7 \mathrm{~S}$ ratio for both locations was 1.28 and 2.00 , respectively. In Rio Verde, concentrations of both protein fractions, beta-conglicinin and glycinin, were reduced (Table 4). The highest values for glycinin, acidic and basic subunits were observed for sowings in Senador Canedo. The inverse relationship between total $7 \mathrm{~S}$ and $11 \mathrm{~S} / 7 \mathrm{~S}$ ratio can be observed from data of Anápolis, which exhibited lower concentrations of beta-conglycinin subunits and higher concentrations of glycinin.

Genetic variability was observed among Brazilian soybean cultivars, as well as effects of the environment on total and individual protein fractions and their subunits. However, in the present study, due to lack of specific observations on temperatures, precipitation and light incidence, it was not possible to identify which environmental component had stronger impact on concentration

Table 4. Glycinin and beta-conglycinin protein fraction composition(\%), in seeds of soybean cultivar MG/BRS 46 (Conquista), sown in 16 locations of Goiás (GO) and Minas Gerais (MG) states in Central Brazil. Soybean season 2001/2002 ${ }^{(1)}$.

\begin{tabular}{|c|c|c|c|c|c|c|c|c|c|}
\hline \multirow[t]{2}{*}{ Locations } & \multicolumn{4}{|c|}{ Beta-Conglycinin (7S) } & \multicolumn{3}{|c|}{ Glycinin (11S) } & \multirow{2}{*}{$\begin{array}{c}11 \mathrm{~S} / 7 \mathrm{~S} \\
\text { Ratio }\end{array}$} & \multirow{2}{*}{$\begin{array}{c}\text { Total } \\
7 \mathrm{~S}+11 \mathrm{~S}\end{array}$} \\
\hline & Alpha' & Alpha & Beta & Total & Acidic & Basic & Total & & \\
\hline Cristalina 1 & $7.08 \mathrm{a}$ & $8.57 \mathrm{a}$ & $9.21 \mathrm{a}$ & $24.86 a$ & $18.77 \mathrm{ab}$ & $15.78 \mathrm{bc}$ & $34.55 \mathrm{ab}$ & $1.96 \mathrm{bc}$ & $59.42 \mathrm{a}$ \\
\hline Iraí de Minas & $5.44 \mathrm{bc}$ & $8.28 \mathrm{ab}$ & $8.48 \mathrm{ab}$ & $22.21 b$ & $17.02 \mathrm{bc}$ & $13.76 \mathrm{~cd}$ & $30.79 b c$ & $1.38 \mathrm{~g}$ & $53.00 \mathrm{abcd}$ \\
\hline Sacramento & $6.18 \mathrm{ab}$ & $8.37 \mathrm{ab}$ & $6.31 \mathrm{~cd}$ & $20.87 \mathrm{bc}$ & $18.79 \mathrm{ab}$ & $15.74 b c$ & $34.52 \mathrm{ab}$ & $1.65 \mathrm{f}$ & $55.39 \mathrm{abc}$ \\
\hline Chapadão do Céu & $5.16 \mathrm{bcd}$ & $6.47 \mathrm{efg}$ & $9.00 \mathrm{a}$ & $20.63 \mathrm{bcd}$ & $13.99 \mathrm{~cd}$ & $12.41 \mathrm{~d}$ & $26.40 \mathrm{c}$ & $1.28 \mathrm{~g}$ & $47.04 d$ \\
\hline Uberlândia & $6.18 \mathrm{ab}$ & 7.70abcd & $6.42 \mathrm{~cd}$ & $20.30 \mathrm{bcd}$ & $19.58 \mathrm{ab}$ & $15.74 b c$ & $35.32 \mathrm{ab}$ & $1.73 \mathrm{def}$ & $55.63 \mathrm{abc}$ \\
\hline Buritis & $5.47 \mathrm{bc}$ & $7.90 \mathrm{abc}$ & $6.53 c$ & $19.90 \mathrm{bcde}$ & $18.04 \mathrm{abc}$ & $13.63 \mathrm{~cd}$ & $31.68 \mathrm{bc}$ & $1.59 \mathrm{f}$ & $51.58 \mathrm{bcd}$ \\
\hline Senador Canedo & $6.35 \mathrm{ab}$ & $8.22 \mathrm{ab}$ & 5.10efgh & 19.68bcdef & $20.52 \mathrm{a}$ & $18.87 \mathrm{a}$ & $39.39 \mathrm{a}$ & $2.00 \mathrm{abc}$ & $59.07 \mathrm{ab}$ \\
\hline Luziânia & $4.50 \mathrm{~cd}$ & $7.12 \mathrm{cde}$ & $7.95 b$ & $19.58 \mathrm{bcdef}$ & $17.49 \mathrm{bc}$ & $15.56 \mathrm{bc}$ & $33.05 \mathrm{~b}$ & $1.69 \mathrm{ef}$ & $52.62 \mathrm{abcd}$ \\
\hline Uberaba & $6.00 \mathrm{ab}$ & $7.50 \mathrm{bcd}$ & $5.54 \mathrm{ef}$ & $19.05 \mathrm{defg}$ & $19.30 \mathrm{ab}$ & $16.24 \mathrm{abc}$ & $35.55 \mathrm{ab}$ & $1.86 \mathrm{~cd}$ & $54.60 \mathrm{abcd}$ \\
\hline Conquista & $5.80 \mathrm{abc}$ & $7.00 \mathrm{cde}$ & $5.33 \mathrm{efg}$ & 18.13defgh & $17.78 \mathrm{abc}$ & $15.94 \mathrm{abc}$ & $33.72 b$ & $1.86 \mathrm{cde}$ & $51.86 \mathrm{abcd}$ \\
\hline Alvorada & $4.93 \mathrm{bcd}$ & $8.05 \mathrm{ab}$ & $4.63 \mathrm{gh}$ & $17.61 \mathrm{efgh}$ & $17.87 \mathrm{abc}$ & $17.01 \mathrm{ab}$ & $34.88 \mathrm{ab}$ & $1.98 \mathrm{bc}$ & $52.50 \mathrm{abcd}$ \\
\hline Cristalina 2 & $5.84 \mathrm{abc}$ & $6.79 \mathrm{def}$ & $4.52 \mathrm{~h}$ & $17.15 \mathrm{fgh}$ & $18.62 \mathrm{ab}$ & $15.17 \mathrm{bcd}$ & $33.79 b$ & $1.39 \mathrm{~g}$ & $50.94 \mathrm{~cd}$ \\
\hline S.M. do Passa Quatro & $4.91 \mathrm{bcd}$ & $5.78 \mathrm{~g}$ & $5.75 \mathrm{de}$ & $16.44 \mathrm{gh}$ & $16.85 \mathrm{bc}$ & $14.87 \mathrm{bcd}$ & $31.72 b c$ & $1.92 \mathrm{c}$ & $48.16 \mathrm{~cd}$ \\
\hline Cerrados & $5.48 \mathrm{bc}$ & $6.57 \mathrm{efg}$ & $4.37 \mathrm{~h}$ & $16.44 \mathrm{gh}$ & $17.95 \mathrm{abc}$ & $16.95 \mathrm{ab}$ & $34.90 \mathrm{ab}$ & $2.12 \mathrm{ab}$ & $51.34 \mathrm{bcd}$ \\
\hline Anápolis & $5.50 \mathrm{bc}$ & $6.01 \mathrm{fg}$ & $4.91 \mathrm{fgh}$ & $16.42 \mathrm{gh}$ & $18.85 \mathrm{ab}$ & $16.58 \mathrm{abc}$ & $35.44 \mathrm{ab}$ & $2.16 \mathrm{a}$ & $51.85 \mathrm{abcd}$ \\
\hline Rio Verde & $3.86 \mathrm{~d}$ & $5.81 \mathrm{~g}$ & $6.61 \mathrm{c}$ & $16.28 \mathrm{gh}$ & $15.84 \mathrm{~cd}$ & $14.87 \mathrm{bcd}$ & $30.71 b c$ & $1.89 \mathrm{~cd}$ & $46.99 \mathrm{~d}$ \\
\hline
\end{tabular}

${ }^{(1)}$ Means followed by the same letters, in the columns, do not differ by Tukey test, at $5 \%$ of probability. 
of the protein components. Genotype x environment interactions should be considered in future studies.

\section{Conclusions}

1. Among Brazilian soybean cultivars, there are genetic differences for contents of $11 \mathrm{~S}$ (glycinin) and $7 \mathrm{~S}$ (beta-conglycinin) protein fractions, and there is a good possibility of breeding for individual protein fractions, and their subunits, without affecting protein content.

2. 'MS/BRS 169' (Bacuri) presents the better protein quality with the highest $11 \mathrm{~S} / 7 \mathrm{~S}$ ratio.

3 . Sowing locations affect concentration of these compounds.

\section{Acknowledgements}

To Emidio Bonato (Embrapa Trigo), Maurício Assunção (Centro Tecnológico para Pesquisas Agropecuárias), Neylson Eustaquio Arantes (Embrapa Soja), Orival Gastão Menosso (Embrapa Soja), Plinio Itamar de Mello de Souza (Embrapa Cerrados), Ricardo Montalván (Embrapa Soja, Balsas), and José Marcos Gontijo Mandarino (Embrapa Soja), for providing samples for the experiments; to Donna Thomas and Richard Hens (United States Department of Agriculture, Agricultural Research Service, National Center for Agricultural Utilization Research), for preparing samples; to Maria Cristina Neves de Oliveira (Embrapa Soja), for the statistical analysis.

\section{References}

BRADFORD, M.M. A rapid and sensitive method for the quantitation of microgram quantities for protein utilizing the principle of protein-dye binding. Analytical Biochemistry, v.72, p.248-254, 1976.

CHUA, N.H. Eletrophoretic analysis of chloroplast proteins. Methods in Enzymology, v.69, p.434-446, 1980.

DURANTI, M.; LOVATI, M.R.; DANI, V.; BARBIROLI, A.; SCARAFONI, A.; CASTIGLIONI, S.; PONZONE, C.; MORAZZONI, P. The $\alpha$ ' subunit from soybean 7S globulin lowers plasma lipids and upregulates liver B-VLDL receptors in rats fed a hypercholesterolemic diet. Journal of Nutrition, v.134, p.1334-1339, 2004.

FEHR, W.R.; HOECK, J.A.; JOHNSON, S.L.; MURPHY, P.A.; NOTT, J.D.; PADILLA, G.I.; WELKE, G.A. Genotype and environment influence on protein components of soybean. Crop Science, v.43, p.511-514, 2003.
FUKUSHIMA, D. Recent progress in research and technology on soybeans. Food Science and Technology Research, v.7, p.8-16, 2001.

HARADA, K.; TOYOKAWA, Y.; KITAMURA, K. Genetic analysis of the most acidic $11 \mathrm{~S}$ globulin subunit and related characters in soybean seeds. Japanese Journal of Breeding, v.33, p.23-30, 1983.

HELMS, T.C.; CAI, T.D.; CHANG, K.C.; ENZ, J.W. Tofu characteristics influenced by soybean crop year and location. 1998. Available at: <http://www.ag.ndsu.nodak.edu/ ndagres/fall98/ar31198.htm>. Accessed on: 3 Nov. 2006.

JOHNSON, R.A.; WICHERN, D.W. Applied multivariate statistical analysis. 6.ed. New Jersey: Prentice Hall, 2007. 800 p.

KITAMURA, K.; KAIZUMA, N. Mutant strains with low level of subunits of $7 \mathrm{~S}$ globulin in soybean (Glycine max Merr.) seed. Japanese Journal of Breeding, v.31, p.353-359, 1981.

KITAMURA, K.; TAKAGI, T.; SHIBASKI, K. Subunit structure of soybean 11S globulin. Agricultural and Biological Chemistry, v.41, p.351-356, 1976.

KWANYUEN, P.; PANTALONE, V.R.; BURTON, J.W.; WILSON, R.F. A new approach to genetic alteration of soybean protein composition and quality. Journal of the American Oil Chemists' Society, v.74, p.983-987, 1997.

LOVATI, M.R.; MANZONI, C.; GIANAZZA, E.; SIRTORI, C.R. Soybean protein products as regulators of liver lowdensity lipoprotein receptors. I. Identification of active ß-conglycinin subunits. Journal of Agricultural and Food Chemistry, v.46, p.2474-2480, 1998.

MILLER, I.; HAYNES, P.; GEMEINER, M.; AEBERSOLD, R.; MANZONI, C.; LOVATI, M.R.; VIGNATI, M.; EBERINI, I.; GIANAZZA, E. Proteins of rat serum. II. Influence of some biological parameters of the two-dimensional electrophoresis pattern. Electrophoresis, v.19, p.1493-1500, 1998.

MORAES, R.M.A. de; JOSÉ, I.C.; RAMOS, F.G.; BARROS, E.G. de; MOREIRA, M.A. Caracterização bioquímica de linhagens de soja com alto teor de proteína. Pesquisa Agropecuária Brasileira, v.41, p.725-729, 2006.

MURPHY, P.A.; RESSURECCION, A.P. Varietal and environmental differences in soybean glycinin and $\beta$-conglycinin content. Journal of Agricultural and Food Chemistry, v.32, p.911-915, 1984.

OGAWA, T.; TAYAMA, E.; KITAMURA, K.; KAIZUMA, $\mathrm{N}$. Genetic improvement of seed storage proteins using three variant alleles of $7 \mathrm{~S}$ globulin subunits in soybean (Glycine $\max$ L.). Japanese Journal of Breeding, v.39, p.137-147, 1989.

SAIO, K.; KAMIYA, M.; WATANABE, T. Food processing characteristics of soybean $11 \mathrm{~S}$ and $7 \mathrm{~S}$ proteins. Part I. Effect of difference of protein component among soybean varieties on formation of tofu-curd. Agricultural and Biological Chemistry, v.33, p.1301-1308, 1969.

SCOTT, A.J.; KNOTT, M. A cluster analysis method for grouping means in the analysis of variance. Biometrics, v.30, p.507-512, 1974. 
STASWICK, P.E.; HERMODSON, M.A.; NIELSEN, N.C. The amino acid sequence of the A2B 1 a subunit of glycinin. Journal of Biological Chemistry, v.259, p.13424-13430, 1984.

TAKAHASHI, M.; HAJIKA, M.; MATSUNAGA, R.; KOMATSU, K.; OBATA, A.; KANAGAE, R. Breeding soybean variety lacking $\beta$-conglycinin by introduction of $S c g$ gene from wild soybean. In: INTERNATIONAL SOYBEAN PROCESSING AND UTILIZATION CONFERENCE, 3., 2000, Tsukuba. Proceedings. Tsukuba: The Japanese Society for Food Science and Technology, 2000. p. $45-46$

TCHIENKOUA, M.; ZECH, W. Statistical analysis of soil variability in a humid forest landscape of Central Cameroon. International Journal of Applied Earth Observation and Geoinformation, v.5, p.69-79, 2004.

THANH, V.H.; SHIBASAKI, K. Beta-conglycinin from soybean proteins. Isolation and immunological and physicochemical properties of the monomeric forms. Biochimica et Biophysica Acta, v.490, p.370-384, 1977.
TSUKADA, Y.; KITAMURA, K.; HARADA, K.; KAIZUMA, $\mathrm{N}$. Genetic analysis of subunits of two major storage proteins ( $\beta$-conglycinin and glycinin) in soybean seed. Japanese Journal of Breeding, v.36, p.390-400, 1986.

USFDA. Food and Drug Administration. Food labeling, health claims, soy protein and coronary heart disease; final rule. Federer Register, v.64, p.57700-57733, 1999.

UTSUMI, S.; MATSUMURA, Y.; MORI, T. Structure-function relationship of soy proteins. In: DAMODARAM, S.; PARAF, A. (Ed.). Food proteins and their applications. New York: Marcel Dekker, 1997. p.257-291.

YAKLICH, R.W. $\beta$-conglycinin and glycinin in high protein soybean seeds. Journal of Agricultural and Food Chemistry, v.49, p.729-735, 2001.

YAMAUCHI, F.; SATO, M.; SATO, W.; KAMATA, Y.; SHIBASAKI, K. Isolation and identification of a new type of $\beta$-conglycinin in soybean globulins. Agricultural and Biological Chemistry, v.45, p.2863-2868, 1981.

Received on June 5, 2008 and accepted on August 19, 2008 